\title{
AN UNSUPERVISED CHANGE DETECTION BASED ON TEST STATISTIC AND KI FROM MULTI-TEMPORAL AND FULL POLARIMETRIC SAR IMAGES
}

\author{
J. Q. Zhao ${ }^{\text {a, J. Yang }}{ }^{\text {a, *, P. X. Li }}{ }^{\text {a, M. Y. Liu }}{ }^{\text {a }}$, Y. M. Shi ${ }^{\text {a }}$ \\ ${ }^{a}$ The State Key Laboratory of Information Engineering in Surveying, Mapping and Remote Sensing of Wuhan University, Wuhan, \\ P. R. China-(masurq, yangj, pxli, amylmy, 2011302590179)@whu.edu.cn
}

Commission VII, WG VII/5

KEY WORDS: Change Detection, Multi-temporal, Synthetic Aperture Radar (SAR), Test Statistic, Kittler and Illingworth (KI)

\begin{abstract}
:
Accurate and timely change detection of Earth's surface features is extremely important for understanding relationships and interactions between people and natural phenomena. Many traditional methods of change detection only use a part of polarization information and the supervised threshold selection. Those methods are insufficiency and time-costing. In this paper, we present a novel unsupervised change-detection method based on quad-polarimetric SAR data and automatic threshold selection to solve the problem of change detection. First, speckle noise is removed for the two registered SAR images. Second, the similarity measure is calculated by the test statistic, and automatic threshold selection of KI is introduced to obtain the change map. The efficiency of the proposed method is demonstrated by the quad-pol SAR images acquired by Radarsat-2 over Wuhan of China
\end{abstract}

\section{INTRODUCTION}

Remotely sensed imagery of a geographical area of interest represents a precious source of information for environmental monitoring applications (e.g., land-use and land-cover dynamic analysis, crop monitoring, and deforestation studies). In particular, the availability of synthetic aperture radar (SAR) data provides great potential from this viewpoint due to the insensitiveness of SAR sensors to atmospheric (in particular, rain and clouds) and sunlight-illumination conditions (Moser, \& Serpico, 2006). In addition, as the space-borne SAR sensors increased, it is more convenient to acquire mass of multitemporal SAR data. In light of these benefits, a number of studies have been performed using multi-temporal SAR over the change detection.

Several approaches have been proposed to deal with change detection based on multi-temporal SAR images. In general, the approach of change detection contains three steps: (1) preprocessing of registration and filtering; (2) calculating change image by similarity measure; (3) obtaining change-detection map. Most of approaches focus on how to estimate the similarity measure of multi-temporal SAR data and to obtain the decision threshold. In the first problem, there have been some methods to solve it. Based on algebraic operation, the difference and ratio (Rignot, \& Van Zyl, 1993) are the traditional methods of change detection. Because of using parts of SAR information, the results of change detection easily appear some detection error. Based on image analysis, PCA (Qiu, 2003) and CVA (Bovolo, \& Bruzzone, 2006) are introduced. However, the computation of the above methods is complicated. Based on classification, the methods of the post-classification comparison (Bruzzone, 1999) and the joint-classification comparison are simple and effective to process the change detection. But the quality of their results critically depends on the accuracy of classification. If the result of classification is not accurate, the result of change detection will have some false detection. Some methods based on statistical probability density function such as Kullback-Leibler (KL) (Inglada, \& Mercier, 2007) and test statistic (Conradsen, 2003) use more SAR information and polarimetric feature of the SAR data. Because of those reasons, the estimation of change detection is more stable. In the second problem, there are two types of method which are supervised and unsupervised respectively (Bazi, 2005). Supervised threshold selection is manual operations, and it typically turn out to be time consuming. Unsupervised technique of threshold selection is used to solve this problem. And the automatizing method that integrates image with a generalization of the Kittler and Illingworth (K\&I) minimum-error thresholding algorithm (Kittler, \& Illingworth, 1986) is introduced. This method is proved to be effective in decision threshold (Bazi, 2005; Moser, \& Serpico, 2006).

In this paper, we present a novel unsupervised change-detection method based on quad-polarimetric SAR data and automatic threshold selection. Using all information of PolSAR data, we use the method of test statistic to test for equality of two complex Wishart matrices. Using the statistical property to judge the change is more stable and effective. Although comparison pixels have different grey value, the same places have the similar probability density function. We use test statistic to obtain the similarity measure of multi-temporal SAR images. Using the thresholding criterion of KI, we obtain the decision threshold automatically. Finally, this threshold is used to divide the image of similarity measure in two parts, and the map of change-detection is obtained.

In section 2, the proposed change-detection methodology is derived, and test statistic and KI are introduced. Section 3 reports the results of the proposed approach on the multitemporal SAR data over WuHan. Finally, conclusions are drawn in section 4 . 


\section{METHOD}

\subsection{The distribution of SAR image}

A p-dimensional random complex vector $\mathrm{Z}$ satisfies with a complex multivariate normal distribution as follow:

$$
Z=\left[\mathrm{Z}_{1} \ldots \mathrm{Z}_{p}\right] \in N_{c}(0, \Sigma)
$$

And its frequency function presents:

$$
f(z)=\frac{1}{\pi^{p}|\Sigma|} \exp \left\{-\operatorname{tr}\left[\Sigma^{-1} z z^{* T}\right]\right\}
$$

Where $|\bullet|$ denotes the determinant; tr denotes the trace of a matrix; and ${ }^{* T}$ denotes complex conjugation $\left({ }^{*}\right)$ and transpose $\left({ }^{T}\right)$.

And a Hermitian positive definite random $\mathrm{p}^{*} \mathrm{p}$ matrix X satisfies with a complex Wishart distribution as follow:

$$
X \in W_{c}(p, \mathrm{n}, \Sigma)
$$

And its frequency function presents:

$$
w(x)=\frac{1}{\Gamma_{p}(n)} \frac{1}{|\Sigma|^{n}}|x|^{n-p} \exp \left\{-\operatorname{tr}\left[\Sigma^{-1} w\right]\right\}
$$

Where:

$$
\Gamma_{p}(n)=\pi^{p(p-1) / 2} \prod_{j=1}^{p} \Gamma(n-j+1)
$$

The $\mathrm{w}$ is positive definite. And if $\mathrm{X}$ and $\mathrm{Y}$ follow the complex Wishart distribution and are independent, their sum follows complex Wishart distribution.

$$
S=X+Y \in W_{c}(p, n+m, \Sigma)
$$

\subsection{Test for Equality of Two Complex Wishart Matrices}

In process of change detection, we always distinguish the image in changed part and un-changed part based on similarity of multi-temporal data, and using the assumption of null hypothesis and alternative hypothesis replaces the un-change and change. We consider the null hypothesis $H_{0}: \Sigma_{x}=\Sigma_{y}$ means that two matrices are equal and deem that is un-change. We consider the alternative hypothesis $H_{1}: \Sigma_{x} \neq \Sigma_{y}$ means that two matrices are difference and deem that is change.

The likelihood ratio presents as follow,

$$
Q=\frac{L(\bar{\Sigma})}{L_{x}\left(\bar{\Sigma}_{x}\right) L_{y}\left(\bar{\Sigma}_{y}\right)}
$$

Here:

$$
L_{x}\left(\bar{\Sigma}_{x}\right)=\frac{1}{\Gamma_{p}(n)}\left|\frac{1}{n} X\right|^{-n}|X|^{n-p} \exp \{-n t r I\}
$$

Putting the formula (8) into formula (7), the desired likelihoodratio test statistic presents as follow:

$$
Q=\frac{(n+m)^{p(n+m)}}{n^{p n} m^{p m}} \frac{|X|^{n}|Y|^{m}}{|X+Y|^{n+m}}
$$

The typically case for change detection is $n=m$, we get $-2 \rho \ln Q$ as follow:

$$
-2 \rho \ln Q=-2 \rho n(2 p \ln 2+\ln |X|+\ln |Y|-2 \ln |X+Y|)
$$

Here:

$$
\rho=1-\frac{2 p^{2}-1}{6 p}\left(\frac{1}{n}+\frac{1}{m}-\frac{1}{n+m}\right)
$$

The formula (10) uses to generate the similarity measure based on multi-temporal SAR images.

\section{$2.3 \mathrm{~K} \& I$}

From the result of test-statistic, we obtain the single image of similarity measure. We assume that grey level values of an image are $\mathrm{g}$ from the interval of $0 \sim \mathrm{n}$. And each of the two components $p(g \mid i)$ of the changed and unchanged pixels follow generalized Gaussian distribution (GGD) with mean $\mu_{i}$, standard deviation $\sigma_{i}$ and prior probability $p_{i}$ as follows:

$$
\begin{gathered}
p_{i}(T)=\sum_{g=a}^{b} h(g) \\
\mu_{i}(T)=\sum_{g=a}^{b} h(g) \mathrm{g} / p_{i}(T) \\
\sigma_{i}^{2}(T)=\sum_{g=a}^{b}\left\{g-u_{i}(T)\right\}^{2} h(g) / p_{i}(T)
\end{gathered}
$$

Where,

$$
\begin{gathered}
a=\left\{\begin{array}{l}
0, i=1 \\
T+1, \mathrm{i}=2
\end{array}\right. \\
b=\left\{\begin{array}{l}
T, i=1 \\
n, \mathrm{i}=2
\end{array}\right.
\end{gathered}
$$

Using the model of $h(g \mid i, T)$, the conditional probability $e(g, T)$ can be replaced as follow:

$$
e(g, T)=h(g \mid i, T) p_{i}(T) / h(g)
$$

As $h(g)$ is independent of both $\mathrm{i}$ and $\mathrm{T}$, we can represents $\varepsilon(g, T)$ as follow,

$$
\begin{aligned}
\varepsilon(g, T) & =-2 * \log e(g, T) \\
i & =\left\{\begin{array}{l}
1, g<T \\
2, g>T
\end{array}\right.
\end{aligned}
$$

Which can be considered as an alternative index of correct classification performance. The average performance figure of the whole image can be characterized by the criterion function

$$
J(T)=\sum_{g} h(g) \varepsilon(g, T)
$$

The role of this criterion function in finding the (approximate) minimum error threshold is subtle and the formula shows as follow,

$$
J(r)=\min _{T} J(T)
$$

The criterion $J(T)$ can be expressed as follow:

$$
\begin{aligned}
& J(T)=1+2\left[p_{1}(T) \log \sigma_{1}(T)+p_{2}(T) \log \sigma_{2}(T)\right] \\
& -2\left[p_{1}(T) \log p_{1}(T)+p_{2}(T) \log p_{2}(T)\right]
\end{aligned}
$$

\subsection{The flow of algorithm}

The main procedure of our method is as follows.

1) In the first step, two temporal data of SAR image should be filtered to remove the noise; In general, we choose the refined Lee filtering with a $7 * 7$ window (Lee, 1999). After filtering, two images were registered.

2) In the second step, the formula (10) obtains the similarity measure by test statistic based on multi-temporal polarimetric SAR data. 
3) In the third step, threshold is chosen by KI (formula (22)) in the image of similarity measure, and the changedetection map obtains by this threshold.

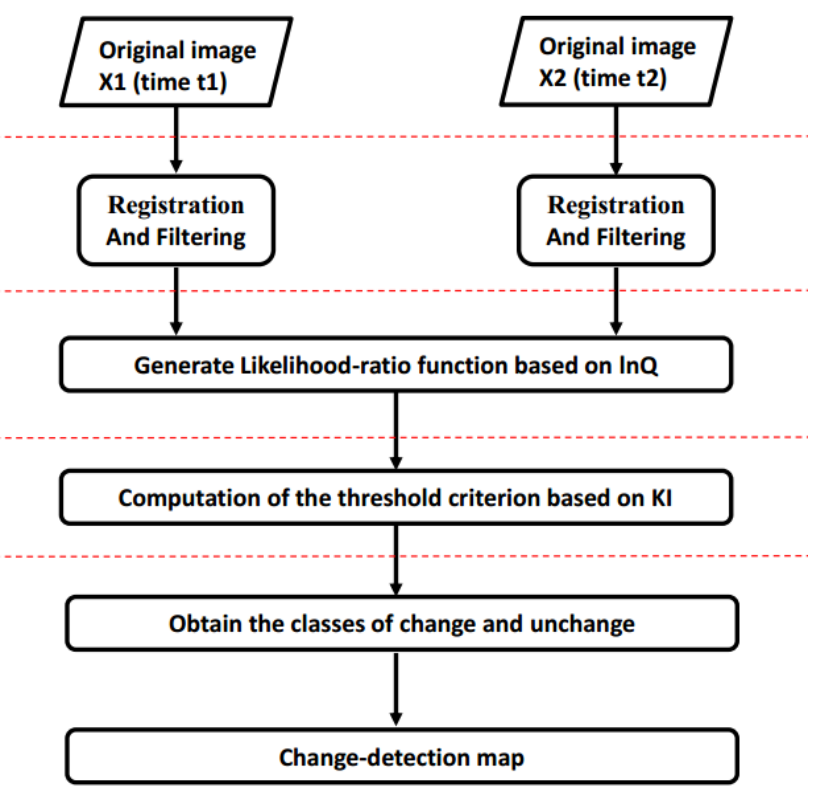

Fig.1 Flow of our method

\section{DATASETS DESCRIPTION AND EXPERIMENTS}

In order to assess the effectiveness of the proposed approach in this section, we used the multi-temporal and quad-pol SAR images over Wuhan and two selected regions of WuHan are considered in the experiments.

\subsection{Datasets}

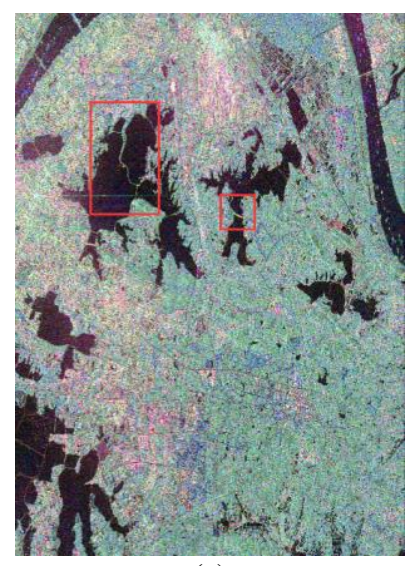

(a)

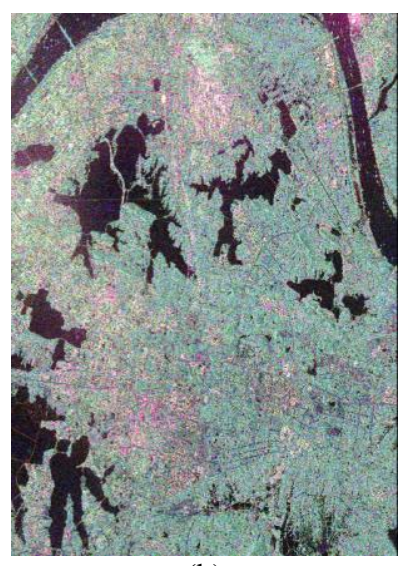

(b)
Fig.2 The Pauli-RGB image of WuHan on (a) December 07, 2011, and (b) June 25, 2015.

In our experiment, we use the data of Wuhan acquired from RADARSAT-2 on December 07, 2011 and June 25, 2015, respectively. Fig. 2 shows the Pauli-RGB images of two periods. We present two sections $(400 * 400)$ in those datasets shown in figure 3. Hence, in the first areas of section, the Eastlake Ave was built after 2011. And in the second areas of section, because of developed of urbanization, lakes, cities and bridge had been changed after 2011. The result of change detection of those two parts will demonstrate the effective of our method.

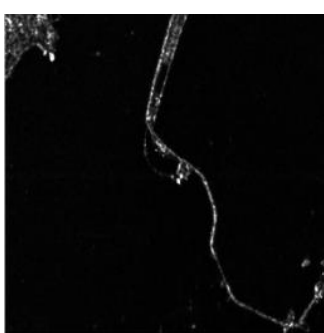

(a)

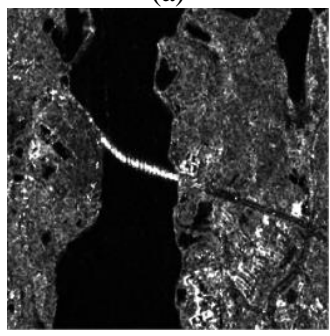

(c)

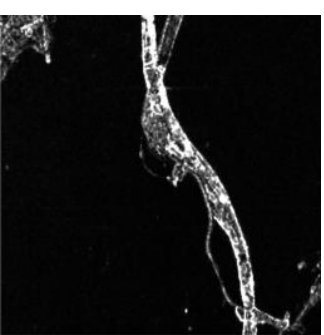

(b)

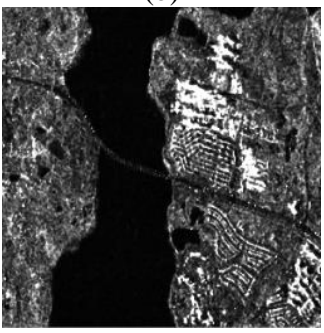

(d)
Fig.3 The span of the First region of WuHan on (a) December 07, 2011 and (b) June 25, 2015, and the Second region on (c) December 07, 2011 and (d) June 25, 2015.

\subsection{Experiments}

In order to test the effectiveness of our method, we choose PCA, JCC and proposed method to experiment, respectively. The multi-temporal SAR data are shown in the Fig.3. In the Fig.3 (a) and (b), the Eastlake Ave has been built. In the Fig.3 (c) and (d), lakes have been replaced by lands, and bridge has been built and at el. As the part of change is smaller than unchanged part, PCA divide the image into the principal component (unchanged) and the minor component (changed), and the results of PCA are shown in the Fig.4 (a) and (b). There have many separate points in the block region, such as the new Eastlake Ave and some parts of yellow frames in the Fig.4 (b). Because of PCA based on image analysis, it uses the single pixel to compare, and many separate changed points can be found. As only using the information of single band, PCA cannot use the full information of the SAR image. JCC is the method based on similarity measure and classification. The multi-temporal and fullpolarimetric SAR data are used, and the results of JCC are shown in the Fig.4 (c) and (d). There have good performances for some target points, but still have many fractured in the block region. Because of JCC based on classification, the result of change detection depends on the accuracy of the classification and the rule of similarity measure. So the good classifiers should be choose, and it will be time-consuming. Both PCA and JCC have some mistakes in the change detection. Such as another tunnel already exist in 2011, but PCA and JCC consider it changed. Those mistakes also appear in the areas of city at image top left. Our method based on test-statistic and KI uses the quad-polarimetric information of SAR image and automatic threshold selection. Firstly, we use the statistical property of SAR image and use the Wishart distribution. Using the statistical property to judge the change is more stable and effective than using the single pixels. Although comparison pixels have different grey value, the same places have the similar probability density function. Using the Wishart distribution is fit for the properties of polarimetric SAR image. Secondly, we use the Kittler and Illingworth minimum-error thresholding algorithm to automatic the threshold. This method chosen the threshold based on the role of criterion function in finding the minimum error. It is subtle and effective. The result shows in Fig.4 (e). The detail remains good and target points 
have good preserved. Moreover, another tunnel considers the unchanged part. Because of using the value of Statistics, the estimate value more stable than only the value of pixels, and the detection is more robustness and reasonable. The complex scene has been shown in the Fig.3 (c) and (d), the result of PCA, JCC, and our method is shown in Fig.4, respectively. In the regions of lake, change detection based on our method has better performance than PCA and JCC, because of the block areas detected. And in those regions, the result is continuous and more smoothly. In the regions of cities, comparing with the dim detection of contour based on PCA and JCC, our method remains the change of contour of cities. In the region of new built bridge, comparing with the fracture phenomenon in PCA and JCC, the result of our method is continuous and more smoothly. All those contrast experiments verifies the robust and effectiveness of the new algorithm.

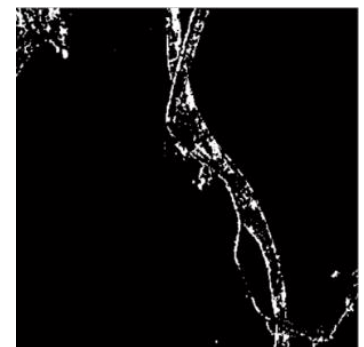

(a)

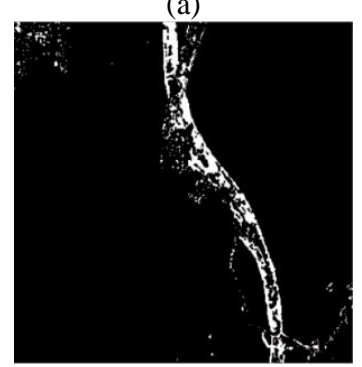

(c)

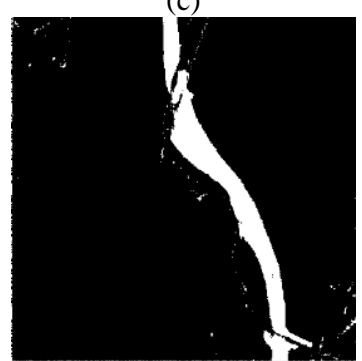

(e)

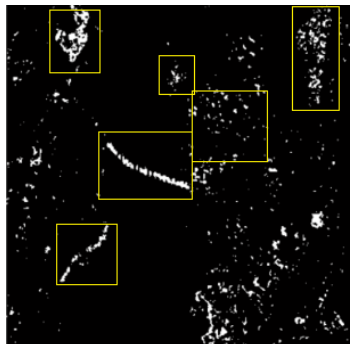

(b)

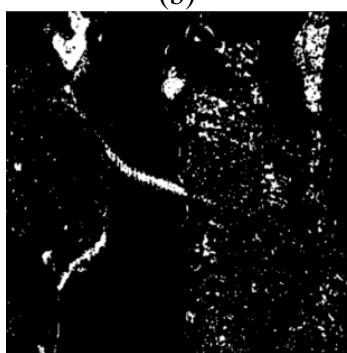

(d)

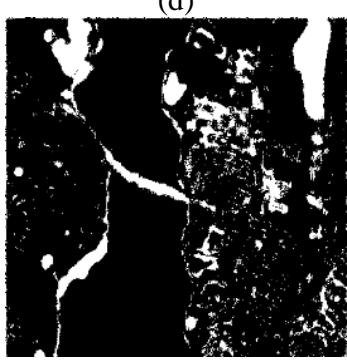

(f)
Fig.4 The results derived from PCA of the (a) first region in WuHan and (b) second region. The result derived from JCC of the (c) first region in WuHan and (d) second region. The result derived from proposed method of the (e) first region in WuHan and (f) second region.

\section{CONCLUSION}

A novel method of change detection based on Multi-temporal and PolSAR Image is presented. We present a novel unsupervised change-detection method based on quadpolarimetric SAR data and automatic threshold selection to solve the problem of similarity measure and threshold selection in change detection. In fully polarimetric SAR data, each pixel denoted by a $3 * 3$ Hermitian positive definite matrix follows a complex Wishart distribution. Based on this distribution, we choose the method of test statistic based on likelihood-ratio to obtain the measure similarity. The proposed approach produces change-detection map according to automatic threshold selection based on Kittler and Illingworth (KI) minimum-error thresholding criterion. Using the fully polarimetric SAR data, we use the more information than SAR amplitude or span imagery. The method of test statistic has good performance on comparing two matrixes followed complex Wishart distribution and obtains good result of change image. Using the KI thresholding criterion, we can automatically classify the change image into two classes, changed class and unchanged class. The efficiency of the proposed method is demonstrated by the quadpol SAR images acquired by Radarsat- 2 over Wuhan of China. The result of two regions is smoothness and integrity, and through comparison experiments of PCA, JCC above, we discover that our method has good performance on detail preserving such as point target and line target in Eastlake and area target in urban, and accurate discover the change regions of bridges, lakes, urban, et al.

\section{ACKNOWLEDGEMENTS}

This research was Funded by National Natural Science Foundation of China under Grant 61371199, 41501382, 91438203; Public Welfare Project of Surveying and Mapping under Grant 201412002; Hubei Provincial Natural Science Foundation of China under Grant 2015CFB328.

\section{REFERENCES}

Bazi, Y., Bruzzone, L., \& Melgani, F. (2005). An unsupervised approach based on the generalized Gaussian model to automatic change detection in multitemporal SAR images. IEEE Transactions on Geoscience and Remote Sensing, 43(4), 874887.

Bovolo, F., \& Bruzzone, L. (2006). A Novel Theoretical Framework for Unsupervised Change Detection Based on CVA in Polar Domain. IEEE International Conference on Geoscience and Remote Sensing Symposium, 2006.

Bruzzone, L., Prieto, D. F., \& Serpico, S. B. (1999). A neuralstatistical approach to multitemporal and multisource remotesensing image classification. IEEE Transactions on Geoscience and Remote Sensing, 37(3), 1350-1359.

Conradsen, K., Nielsen, A. A., Schou, J., \& Skriver, H. (2003). A test statistic in the complex Wishart distribution and its application to change detection in polarimetric SAR data. IEEE Transactions on Geoscience and Remote Sensing, 41(1), 4-19.

Inglada, J., \& Mercier, G. (2007). A new statistical similarity measure for change detection in multitemporal SAR images and its extension to multiscale change analysis. IEEE Transactions on Geoscience and Remote Sensing, 45(5), 1432-1445.

Kittler, J., \& Illingworth, J. (1986). Minimum error thresholding. Pattern recognition, 19(1), 41-47.

Lee, J. S., Grunes, M. R., \& De Grandi, G. (1999). Polarimetric SAR speckle filtering and its implication for classification. IEEE Transactions on Geoscience and Remote Sensing, 37(5), 2363-2373.

Moser, G., \& Serpico, S. B. (2006). Generalized minimum-error thresholding for unsupervised change detection from SAR amplitude imagery. IEEE Transactions on Geoscience and Remote Sensing, 44(10), 2972. 
Qiu, B., Prinet, V., Perrier, E., \& Monga, O. (2003). Multiblock PCA method for image change detection. In IEEE 12th International Conference on Image Analysis and Processing, pp. 385-390.

Rignot, E. J., \& Van Zyl, J. J. (1993). Change detection techniques for ERS-1 SAR data. IEEE Transactions on Geoscience and Remote Sensing, 31(4), 896-906. 